\title{
Introduction to the Special Section of the 1st International MetroArcheo Conference 2015
}

\author{
Sabrina Grassini ${ }^{1}$, Alfonso Santoriello ${ }^{2}$ \\ ${ }^{1}$ Department of Applied Science and Technology, Politecnico di Torino, Italy \\ ${ }^{2}$ Dipartimento di Scienze del Patrimonio Culturale, Università di Salerno, Italy
}

Section: EDITORIAL

Citation: Sabrina Grassini, Alfonso Santoriello, Introduction to the Special Section of the 1st International MetroArcheo Conference 2015, Acta IMEKO, vol. 5, no. 2, article 1, September 2016, identifier: IMEKO-ACTA-05 (2016)-02-01

Section Editors: Sabrina Grassini, Politecnico di Torino, Italy; Alfonso Santoriello, Università di Salerno, Italy

Received August 23, 2016; In final form August 23, 2016; Published September 2016

Copyright: (C) 2016 IMEKO. This is an open-access article distributed under the terms of the Creative Commons Attribution 3.0 License, which permits unrestricted use, distribution, and reproduction in any medium, provided the original author and source are credited

Corresponding author: Sabrina Grassini, email: sabrina.grassini@polito.it; Alfonso Santoriello, email: asantori@unisa.it

Dear Reader,

this special issue of Acta IMEKO contains a selection of papers presented at the 1st International Conference on Metrology for Archaeology - MetroArcheo2015.

This conference, which took place in Benevento, Italy, on October, 22-23 2015, was a real and concrete opportunity for gathering together science and measurement with the humanistic world, specifically archaeology, with the final aim of encouraging discussion and exchange of knowledge and expertise between worlds still considered, often wrongly, distant and different one from the other.

Archaeology, and more generally the cultural heritage field, in recent decades, has undergone so important and profound changes. For over two centuries, archaeological studies have been mainly focused on collecting information and knowledge on the artistic and monumental production of the ancient world. Today it appears clear that the horizons are greatly expanded, and now encompass renewed complex of interactions between the humanistic world and the so-called "exact sciences". New integrated systems able to provide answers to historiographical questions and face real problems in research, protection, monitoring and exploitation of cultural heritage and archaeological sites by providing useful contributions also in terms of urban and regional development planning, have been developed.

The cooperation among experts of different disciplines often results in a multidisciplinary approach in which the individual disciplines and their instruments, work together, but without a unique methodological plan, with the final result of multiplying the problems, increasing the number of documentary series and juxtapose the obtained data. Vice versa, this cooperation should result in a transdisciplinary vision, i.e. comparison among disciplines, and in the creation of new data that should be the splice among the same disciplines. Transdisciplinarity is not just a question of terminology, it presupposes an open rationality which overcomes the excessive formalism.

From the methodological ad conceptual point of view, this transdisciplinary approach really contributes to increase the interest toward archaeology and cultural heritage, by extending their objectives and enriching their technical and instrumental approaches.

Knowing how to read a heritage context taking advantage of the know-how of different disciplines allows one to have access to knowledge and methods, to propose a global and poliedrical view for the study of anthropogenic phenomena, and to develop tailored strategies for conservation, safeguard and management of tangible and intangible cultural heritage and landscapes. Multiplicity and flexibility in the developed approaches also significantly contribute to explain the traces of mankind and their relation with the environment.

Moreover, it should be pointed out that metrology, the science of measurements, includes all aspects both theoretical and practical with reference to measurements, whatever their uncertainty are, and in whatever fields of science or technology they occur. Consequently, the valorisation, characterisation and preservation of cultural heritage is deeply related to metrological issues for the collection, interpretation and 
validation of data collected with the different analytical, physical-chemical, and mechanical techniques, digital technologies, new ICT tools, etc.

MetroArcheo2015 was particularly satisfactory in the methodological and conceptual aspect above-described. This special issue collects ten papers which give a complete view of the different possibilities offered by such integrated analytical and measurement approaches and well represent the worldwide attendance to the Conference.

The first two papers move from the dating to the "quantification" of time in absolute terms (Desachy), to the different methods and statistical analysis systems (Maspero et al.).

The third and fourth papers highlight examples of contributions of archaeometry. The papers show how, through the analysis of stable isotopes and their comparison with archaeological records and other documentary series, it is possible to obtain consistent results in determining diets and health conditions in large territories and in sites of particular relevance in the Central and Southern Italy (Bondioli et al.) and in the Magna Graecia (Ricci et al.).

Another paper describes the importance of geoarchaeological survey and geophysical data managed by GIS, which gives impetus to studies of the environmental contexts and biosphere, overcoming the limits of traditional archaeology, which generally only takes into consideration the anthropogenic contest (Cozzolino et al.).

The paper of Ullo et al. shows how remote sensing, in the frame of the Sentinel-2 mission (2015), allows following the transformations occurred over time, particularly in vegetation, caused both by natural phenomena and anthropogenic causes.

Eventually, the last four contributions deal with several issues and fundamental aspects of protection, such as preventive archaeology and safeguard, monitoring and enhancement of cultural and archaeological heritage in urban areas or in specific sites. One of the discussed cases is devoted to the radical changes of the city of Naples (Italy) due to the construction of the underground line, which allows to put in evidence the extraordinary stratigraphy of the old city. In this contest the use and the optimization of 3D technology surveying, as well as the necessity of improving its accuracy and usability, are fundamental for collecting reliable data (Fregonese et al.).

3D technologies and photogrammetric solutions are discussed in the paper of Limongiello et al. for the documentation and survey of narrow spaces in the "Villa di Giulia Felice" in Pompeii (Italy) for the relief and representation of the monuments with the final aim of monitoring their long-time conservation state.

Another example of the potentialities of three-dimensional techniques for collecting information and reading important details on monuments is provided by the research conducted on the important Harkhuf Tomb in Egypt, inscribed with hieroglyphic texts. The paper of Angelini et al. discusses the methodological approach, the technique and the results obtained thanks to the development of a mathematical model, which provides important information for the safeguard of this important witness of mankind culture.

Eventually, the last paper deals with the study of urban archaeological sites and geophysical prospecting in urban areas, discussing the case studies of the Palatine Hill and the St. John Lateran Basilica in Rome (Piro et al.) highlighting how these non invasive approaches are particularly suitable especially in the presence of archaeological structures built on natural soils.

Our hope is that these contributions are able to highlight the importance of the synergy among the different heritage actors and disciplines, which provides important tools and measuring approaching for the monitoring, preservation, management and safeguard of cultural heritage assets.

Last but not least, it is our sincere hope that this special issue gives the reader new points of interest for metrology and measurement science applied to cultural heritage taking also into account their fundamental role in our society.

Hope you will have an interesting reading!

Sabrina and Alfonso 